This accepted author manuscript is copyrighted and published by Elsevier. It is posted here by agreement between Elsevier and MTA. The definitive version of the text was subsequently published in 2015 Mol. Immunol. 65, 398-405. doi:10.1016/j.molimm.2015.02.014. Available under license $C C-B Y-N C-N D$.

\title{
MASP-1 of the complement system promotes clotting via prothrombin activation
}

\author{
Lorenz Jenny $^{\mathrm{a}, \mathrm{b}}$, József Dobóc ${ }^{\mathrm{c}}$ Péter Gál ${ }^{\mathrm{c}}$, Verena Schroeder ${ }^{\mathrm{a}, \mathrm{b}}$ \\ a University Clinic of Haematology, Haemostasis Research Laboratory, University Hospital Bern, \\ 3010 Bern, Switzerland \\ ${ }^{\mathrm{b}}$ Department of Clinical Research, University of Bern, Bern, Switzerland; \\ ${ }^{\mathrm{c}}$ Institute of Enzymology, Research Centre for Natural Sciences, Hungarian Academy of Sciences, \\ Magyar tudósok krt 2, H-1113 Budapest, Hungary
}

Corresponding author:

Verena Schroeder, PhD

University Clinic of Haematology

Haemostasis Research Laboratory

University Hospital Inselspital

3010 Bern, Switzerland

Tel. +4131632 9618, Fax +4131632 1882

E-mail verena.schroeder@insel.ch 


\section{Abbreviations}

CAPS, N-cyclohexyl-3-aminopropanesulfonic acid; CFT, clot formation time; CT, clotting time; FXa, activated factor X; FXIII, factor XIII; MAp, MBL-associated protein; MASP, mannan-binding lectinassociated serine protease; MBL, mannose binding lectin; MCF, maximum clot firmness; MES, 2(N-Morpholino)- ethanesulfonic acid; PAR4, protease activated receptor 4; PPP, platelet-poor plasma; PT-DP, Prothrombin-depleted plasma; PVDF, polyvinylidene difluoride; rMASP-1cf, MASP-1 catalytic fragment; SGMI, Schistocerca gregaria protease inhibitor (SGPI)-based MASPinhibitor; WB, whole blood.

\section{Abstract}

Mannan-binding lectin-associated serine protease-1 (MASP-1), a protein of the complement lectin pathway, resembles thrombin in terms of structural features and substrate specificity, and it has been shown to activate coagulation factors. Here we studied the effects of MASP-1 on clot formation in whole blood (WB) and platelet-poor plasma (PPP) by thrombelastography and further elucidated the underlying mechanism. Cleavage of prothrombin by MASP-1 was investigated by SDS-PAGE and N-terminal sequencing of cleavage products. Addition of MASP-1 or thrombin to WB and PPP shortened the clotting time and clot formation time significantly compared to recalcified-only samples. The combination of MASP-1 and thrombin had additive effects. In a purified system, MASP-1 was able to induce clotting only in presence of prothrombin. Analysis of MASP-1-digested prothrombin confirmed that MASP-1 cleaves prothrombin at three cleavage sites. In conclusion, we have shown that MASP-1 is able to induce and promote clot formation measured in a global setting using the technique of thrombelastography. We further confirmed that MASP-1-induced clotting is dependent on prothrombin. Finally, we have demonstrated that MASP1 cleaves prothrombin and identified its cleavage sites, suggesting that MASP-1 gives rise to an alternative active form of thrombin by cleaving at the cleavage site R393.

\section{Keywords}

Coagulation; complement system; mannan-binding lectin-associated serine protease; MASP-1; prothrombin; thrombelastography 


\section{Introduction}

The complement system is an integral part of the innate immune system and serves to target, opsonize and eliminate pathogens and foreign bodies from the circulation. More than 30 plasma and membrane-bound proteins are involved in this process which can be subdivided into three different pathways: the lectin, the alternative and the classical pathway. Upon recognition of a target, all three pathways start a proteolytic cascade that leads to the formation of a C3 convertase complex and ultimately ends in clearance of the target (Mayilyan et al., 2008; Ricklin et al., 2010). Many intermediates of these pathways have additional properties, e.g. recruitment of inflammatory cells or crosstalk with the coagulation system. Both the complement and the coagulation cascade originate from the same ancestral pathways, they comprise several serine proteases with common structural characteristics, and they show a high degree of interaction between specific intermediates (Amara et al., 2010; Krem and Di Cera, 2002). As both systems serve as defense mechanism in an environment with compromised barrier setting, they often activate each other (Oikonomopoulou et al., 2012).

In the lectin pathway, mannose binding lectin $(\mathrm{MBL})$ and ficolins recognize target molecules on foreign and/or altered surfaces and thereby induce activation of the MBL-associated serine proteases (MASPs) MASP-1, MASP-2, and MASP-3 (Krarup et al., 2007). MASPs 1-3 and the enzymatically inactive MBL-associated proteins MAp19 and MAp44 are alternative splicing products from two genes: MASP-1/MASP-3/Map44 and MASP2/MAp19 (Stover et al., 1999). Among MASPs, MASP-2 is the only protease capable to form C3 convertase efficiently by cleaving both C2 and C4. MASP-2 was also shown to cleave prothrombin in a similar way to activated factor X (FXa) and hence to promote clot formation (Krarup et al., 2007; Yongqing et al., 2012). In contrast, the function of MASP-3 remains unclear so far. It is speculated that MAp19 competes with the MASPs for the MBL binding site inhibiting activation of the lectin pathway (Dahl et al., 2001; Yongqing et al., 2012). MAp44 has also been shown to regulate the activity of the lectin pathway (Pavlov et al., 2012). 
The exact role of MASP-1 in the complement system has only recently been revised (reviewed in Dobó et al., 2014). MASP-1 was known to cleave C2 but not C4, making it unable to form C3 convertase efficiently by itself. MASP-1 was therefore considered to have a merely supporting role in MASP-2-mediated activation of the lectin pathway. Latest research, however, indicates a central role for MASP-1 in the early lectin pathway by autoactivation and subsequent activation of MASP-2 and MASP-3, attributing MASP-1 the function of the major or even only initiator of the lectin pathway (Héja et al., 2012a; Megyeri et al., 2013).

Even though MASP-1 belongs to the $\mathrm{C} 1 \mathrm{r} / \mathrm{C} 1 \mathrm{~s} / \mathrm{MASP}$ family of serine proteases, its serine protease domain is structurally more closely related to thrombin and trypsin. This is supported by the fact that MASP-1 is more efficiently inhibited by antithrombin (in the presence of heparin) than by $\mathrm{C} 1$ inhibitor (Gál et al., 2009; Parej et al., 2013). Due to its unusually wide substrate binding groove and the resulting broad substrate specificity - which is atypical among complement proteases MASP-1 is able to interact with various targets outside the complement system. Due to its thrombin-like activity, it is able to cleave fibrinogen and factor XIII (FXIII) (Krarup et al., 2008) and it activates protease activated receptor 4 (PAR4) on endothelial cells (Megyeri et al., 2009); however, its efficacy is significantly lower compared to thrombin. The A-subunit of FXIII and the fibrinogen $\beta$-chain are cleaved in the same manner by MASP-1 as by thrombin, while the fibrinogen a-chain is cleaved differently (Krarup et al., 2008). We have shown that clots produced in plasma in presence of MASP-1 have an altered fibrin structure and presented first evidence that MASP-1 may cleave prothrombin (Hess et al., 2012). First in vivo evidence for MASP-1 involvement in coagulation was obtained from MASP-1 and MBL knockout mice which showed prolonged bleeding time on tail tip excision (Takahashi et al., 2012) and a significant decrease in $\mathrm{FeCl}_{3}$-induced thrombogenesis (La Bonte et al., 2012). Taken together, MASP-1 seems to be a major link between the complement system and coagulation but further work is needed to clarify the exact underlying mechanisms and (patho-) physiological relevance.

The aim of this study was to investigate for the first time whether MASP-1 is able to influence or even induce clot formation in human whole blood and how its effects compare with those of thrombin. We went on to elucidate the underlying mechanisms in plasma and purified systems. In 
particular, the role of prothrombin in MASP-1-mediated clotting and their specific interaction were examined.

\section{Materials and methods}

\subsection{MASP-1 protein}

Large amounts of MASP-1 were required for the thrombelastographic experiments. However, neither recombinant production nor plasma purification of pure full-length MASP-1 in sufficient amounts have been achieved so far. We therefore used the recombinant MASP-1 catalytic fragment (rMASP-1cf) for our experiments. It is a truncated form of MASP-1 consisting of the CCP1-CCP2-SP domains while the N-terminal CUB1-EGF-CUB2 domains are deleted (Dobó et al., 2009). In order to confirm that rMASP-1cf and full-length MASP-1 show the same effects on coagulation, we performed a control experiment with active full-length MASP-1 (a kind gift from Mikkel-Ole Skjoedt, Copenhagen, Denmark).

\subsection{Specific protease inhibitors}

SGMI-2 [Schistocerca gregaria protease inhibitor (SGPI)-based MASP-inhibitor] is a specific inhibitor of MASP-2. It is a small protein inhibitor (MW 4009.5 Da) that was expressed in E.coli as described earlier (Héja et al., 2012b). SGMI-2 was dissolved in water at a concentration of 2.83 $\mathrm{mg} / \mathrm{ml}(706 \mu \mathrm{M})$ and used in the thrombelastographic experiments at a final concentration of 5 $\mu \mathrm{mol} / \mathrm{l}$. Hirudin (Novartis, Basel, Switzerland) specifically binds to the active site and exosite 1 of thrombin species and does not interfere with MASP-1 activity (Parry et al., 1993; Presanis et al., 2003). Hirudin was used in prothrombin cleavage experiments at a final concentration of 600 units/ml.

\subsection{Blood collection and sample preparation}

Whole blood (WB) was taken from healthy volunteers who had not taken any medication for 10 days. All volunteers gave written informed consent. Blood was drawn into Sarstedt Monovette ${ }^{\circledR}$ 
tubes containing $0.106 \mathrm{~mol} / \mathrm{l}$ sodium citrate. Platelet-poor plasma (PPP) was produced by centrifugation at $1513 \mathrm{~g}$ for $20 \mathrm{~min}$. Experiments were carried out within $4 \mathrm{~h}$ after blood sampling.

\subsection{Thrombelastography experiments}

Thrombelastographic measurements were performed in fresh WB and PPP following recalcification (final concentration $12.5 \mathrm{mmol} / \mathrm{l} \mathrm{CaCl}_{2}$ ) and addition of $\mathrm{rMASP}-1 \mathrm{cf}$, thrombin, or a combination of rMASP-1cf and thrombin. The mean MASP-1 plasma concentration is $11 \mu \mathrm{g} / \mathrm{ml}$ (equal to 143 $\mathrm{nmol} / \mathrm{l}$ ) (24). We used rMASP-1cf at final concentrations of 5,10 , and $20 \mu \mathrm{g} / \mathrm{ml}$, corresponding to 110, 220, and $440 \mathrm{nmol} / \mathrm{l}$. Thrombin (Calbiochem, Merck Chemicals, Nottingham, UK) was used at final concentrations of 10,20 , and $40 \mathrm{nmol} / \mathrm{l}$, as $26 \mathrm{nmol} / /$ thrombin is the concentration generated at approximately clot time (Brummel et al., 2002). Experiments were performed as follows: Into a thrombelastography cup, we pipetted $16.9 \mu \mathrm{l}$ of $\mathrm{CaCl}_{2}$ and $5.1 \mu \mathrm{l}$ containing rMASP-1cf and/or thrombin in Tris-buffered saline (TBS, $50 \mathrm{mmol} / \mathrm{l}$ Tris, $100 \mathrm{mmol} / \mathrm{l} \mathrm{NaCl}, \mathrm{pH} \mathrm{7.4).} \mathrm{Upon} \mathrm{addition} \mathrm{of}$ $248 \mu \mathrm{l}$ WB or PPP, the measurement was immediately started on a rotation-thrombelastometry system (ROTEM ${ }^{\circledR}$, Tem International, Munich, Germany) and ran for $1 \mathrm{~h}$. Prothrombin-depleted plasma (PT-DP, Hyphen BioMed, Neuville-sur-Oise, France) was used to test if MASP-1-mediated clotting depended on the presence of prothrombin. A purified system containing $2 \mathrm{mg} / \mathrm{ml}(5.8$ $\mu \mathrm{mol} / \mathrm{l})$ fibrinogen in presence or absence of $100 \mu \mathrm{g} / \mathrm{ml}(1.4 \mu \mathrm{mol} / \mathrm{l})$ prothrombin (both from Hyphen BioMed) in TBS was also used. Measurements using PT-DP or purified proteins were carried out for $3 \mathrm{~h}$ to detect any delayed clot formation. We evaluated the standard ROTEM ${ }^{\circledR}$ parameters clotting time (CT; representing the lag time until the onset of clotting), clot formation time (CFT; representing the duration from onset of clotting until $20 \mathrm{~mm}$ of amplitude are reached), and maximum clot firmness (MCF; a measure of visco-elastic properties of the formed clot). Each measurement was performed in triplicate in WB and PPP samples from two individuals. For each individual a recalcified-only sample without MASP-1 or thrombin served as baseline and the results obtained with MASP-1 and/or thrombin were expressed in percentage relative to the baseline values in order to correct for interindividual variation.

\subsection{Prothrombin cleavage studies}


Prothrombin $(1.7 \mu \mathrm{mol} / \mathrm{l} / 120 \mu \mathrm{g} / \mathrm{ml}$, Hyphen BioMed) and rMASP-1cf $(1.75 \mu \mathrm{mol} / \mathrm{l} / 80 \mu \mathrm{g} / \mathrm{ml})$ were incubated for up to $180 \mathrm{~min}$ at $37^{\circ} \mathrm{C}$ in a final volume of $50 \mu \mathrm{l}$ TBS-Buffer. After incubation, the samples were mixed with 4x NUPAGE ${ }^{\circledR}$ sample buffer (4:1; Life Technologies, Carlsbad, USA), boiled for $10 \mathrm{~min}$ at $70^{\circ} \mathrm{C}$, and loaded onto NUPAGE ${ }^{\circledR}$ precast $4-12 \%$ (w/v) gradient gels. Precision Plus Protein ${ }^{\mathrm{TM}}$ Dual Color Standards (10-250 kDa, Biorad, Hercules, USA) was used as protein marker. MES (2-(N-Morpholino)-ethansulfonsäure, Life Technologies) SDS was used as running buffer. For Western blotting we used a $0.2 \mu \mathrm{m}$ polyvinylidene difluoride (PVDF) membrane and CAPS-buffer (N-cyclohexyl-3-aminopropanesulfonic acid, $0.1 \mathrm{M}, \mathrm{pH} 11.0$ ) (both from Life Technologies). After the transfer the membrane was washed and stained with sulforhodamin (sulforhodamin B $89.5 \mu \mathrm{M}, 30 \%$ methanol $(\mathrm{v} / \mathrm{v}), 0.1 \%$ acetic acid $(\mathrm{v} / \mathrm{v})$ ). Bands were excised and subjected to five cycles of Edman degradation and $\mathrm{N}$-terminal sequencing on a $\mathrm{ABI}$ Procise cLC 494-device (Blue Lion Biotech, Carnation, USA).

\section{Results}

\subsection{Effects of MASP-1 on clot formation studied by thrombelastography}

In WB, MASP-1 showed a dose-dependent reduction in CT as shown in Fig. 1a. Addition of 10 $\mu \mathrm{g} / \mathrm{ml}$ rMASP-1cf shortened the CT by $34 \%$ compared to the recalcified-only sample, while thrombin led to a reduction in CT by approximately $90 \%$. Combination of both enzymes did not lead to additive effects. As shown in Fig. 1b, addition of $10 \mu \mathrm{g} / \mathrm{ml} \mathrm{rMASP}-1 \mathrm{cf}$ to PPP decreased the CT by $15 \%$. Again, thrombin shortened CT significantly more and the combination with rMASP-1cf did not show any additional effect. However, a different picture was observed for CFT. MASP-1 reduced the CFT values of the recalcified sample by $41 \%$ in WB (Fig. 2a) and by $29 \%$ in PPP (Fig. $2 b)$, while thrombin led to a decrease by $47 \%$ in WB and by $38 \%$ in PPP, respectively. The combination of both enzymes clearly showed additive effects on CFT: reduction by $67 \%$ in WB and by $47 \%$ in PPP. MASP-1 showed no consistent and significant effects on MCF (data not shown). The results of the control experiment with full-length MASP-1 in WB and PPP showed reduced CT 
and CFT and were consistent with the findings from the rMASP-1cf experiments (Fig. S1 and Table S1 in the supplementary file).

\subsection{Effects of MASP-1 are prothrombin-dependent}

Based on earlier preliminary results (Hess et al., 2012) we hypothesized that MASP-1 may trigger and/or promote clot formation via activation of prothrombin. We therefore performed thrombelastographic measurements in PT-DP and a purified system in the absence and presence of prothrombin. The results are summarized in Table 1.

In PT-DP, even high concentrations of rMASP-1cf failed to induce clotting, whereas thrombin led to clotting that was delayed compared with normal plasma. When PT-DP was reconstituted with prothrombin, rMASP-1cf was able induce clotting. In a purified system containing fibrinogen and thrombin, fibrin formation occurred, whereas MASP-1 alone failed to trigger fibrin formation. However, when prothrombin was added to the purified system containing fibrinogen and rMASP$1 \mathrm{cf}$, we did observe fibrin formation. These results confirm that MASP-1-induced clot formation relies on the presence of prothrombin.

It has been shown that MASP-1 is the main activator of MASP-2 Heja et al., 2012; Megyeri et al., 2013) and that MASP-2 is able to cleave prothrombin (Krarup et al., 2007). Therefore, we wanted to test whether the effects of MASP-1 on clotting observed in WB and PPP could be due to MASP1-mediated activation of MASP-2 and not due to direct action of MASP-1 itself. Thrombelastographic measurements with rMASP-1cf in WB and PPP were repeated in the presence of the specific MASP-2 inhibitor SGMI-2. Inhibition of MASP-2 did not lead to any changes in CT and CFT (data not shown). These results suggest that MASP-2 is not involved in MASP-1-mediated clotting of WB and PPP.

\subsection{MASP-1 cleaves prothrombin}

Our next aim was to confirm that MASP-1 is able to cleave prothrombin and to identify its cleavage site(s). We incubated prothrombin with rMASP-1cf, stopped the reactions at different time points and separated the resulting fragments by SDS-PAGE (as shown in Fig. 3a). Bands were identified by $\mathrm{N}$-terminal sequencing. Band a) corresponds to uncleaved prothrombin, f) to the MASP-1 heavy 
chain, g) to a degradation product of the MASP-1 heavy chain, and i) to the MASP-1 light chain (also shown in the control gel in Fig. 3b). Digestion by prothrombin in the presence of MASP-1 gave rise to several prothrombin fragments (shown in the first lane per time point): b) corresponds to prethrombin-1 $(=\mathrm{F} 2+\mathrm{LC}+\mathrm{HC}$, arises through cleavage at $\mathrm{R} 155), \mathrm{c})$ to a "meizothrombin analogue" cleaved at R393 (instead of R320 as in FXa-mediated prothrombin cleavage), d) corresponds to prethrombin-2 $(=\mathrm{LC}+\mathrm{HC}$, arises through cleavage at $\mathrm{R} 271)$, e) to the fragment F1.F2 (arises through cleavage at R271), h) to a truncated version of the $\alpha$-thrombin heavy chain (from I321 to R393), and j) to the fragment F1. N-terminal sequencing has confirmed the following cleavage sites and corresponding N-termini of the resulting fragments: R155 (SEGGS), R271 (TATSE) and R393 (NIEKI) (Fig. 4). Some cleavage at R155 to yield prethrombin-1 (band b) was due to autodegradation of prothrombin (Fig. 3b). Over time, in the presence of MASP-1 most of the prothrombin has been processed, after 90 min the "meizothrombin analogue" has almost completely undergone further digestion, whereas prethrombin-2 seemed to accumulate. Band h) corresponding to the truncated thrombin heavy chain started to visibly accumulate after approximately $60 \mathrm{~min}$. A time course digestion with $10 \mathrm{~min}$ steps and SDS-PAGE optimized for band $\mathrm{h}$ ) was able to detect this specific band already after 40 min (data not shown).

FXa cleaves prothrombin at R271 and R320, and the resulting products including $\alpha$-thrombin and meizothrombin are involved in its own back-cleavage (Haynes et al., 2012; Krishnaswamy, 2013) Therefore we tested if similar mechanisms would apply to MASP-1-mediated prothrombin cleavage, i.e. if thrombin species and/or intermediate cleavage products would significantly contribute to prothrombin cleavage in presence of MASP-1. We incubated MASP-1 and prothrombin in the presence of hirudin, a specific thrombin inhibitor which also inhibits active thrombin-like intermediates such as meizothrombin. As shown in Fig. 3a (in the second lane per time point), there were differences compared with the digestion lacking hirudin: prothrombin (band a) decreased more slowly, prethrombin-1 (band b) decreased faster, the "meizothrombin analogue" (band c) accumulated, and hardly any truncated thrombin (band h) and less fragment F1 (band j) could be observed. These results suggest that MASP-1 may trigger initial prothrombin degradation and give rise to active thrombin species and intermediates which then contribute to further processing of prothrombin and intermediate cleavage products. 
We also digested prothrombin with thrombin (Fig. 3b). After 90 min prethrombin-1 (band II) accumulated, showing the preference of thrombin to cleave at R155.

\section{Discussion}

Various interactions between the complement system and blood coagulation have been discovered (Amara et al., 2010). In this context the complement protease MASP-1 has moved into the focus of interest in recent years, however, the relevance of its role in coagulation and the underlying mechanisms are not yet fully understood. Most experiments on MASP-1 and coagulation were conducted in isolated systems. In the present study, we investigated for the first time the effects of MASP-1 on clot formation in whole blood by using the technique of thrombelastograhy which simulates a more global setting.

We show that MASP-1 accelerates clotting times and promotes clot formation in WB and PPP. In addition, MASP-1 and thrombin show an additive effect on CFT which is rather remarkable given that thrombin is much more efficient in cleaving FXIII and fibrinogen than MASP-1 (Krarup et al., 2008). This may suggest that MASP-1 exerts different functions than thrombin and not only mirrors thrombin function in a less efficient way.

The influence of MASP-1 on CFT is more pronounced than its influence on CT. As CT reflects the early onset of clotting and CFT measures the kinetics of later clot formation, our data suggest that MASP-1 affects the later phase of clotting rather than the early phase. This delay in its impact on clotting can be due to its lower efficiency towards substrates (Krarup et al., 2008), due to an indirect effect on clotting or a combination of both. We have previously presented first evidence that MASP-1 may activate prothrombin and thus drive clot formation in an indirect way (Hess et al., 2012). This may explain why the effect of MASP-1 is more distinct in the later phase of clot formation: prothrombin needs to be processed to thrombin by MASP-1 before it can support clot formation, while the direct effects of MASP-1 on FXIII and fibrinogen is most likely too small to lead 
to a visibly faster clot formation. This may also explain why there are additive effects of thrombin and MASP-1 on CFT but not CT: with MASP-1 displaying only a negligible direct effect on clotting it is initially not able to contribute to the immediate strong effect of thrombin. However, in the later phase of clot formation, more and more additional thrombin is generated by MASP-1-mediated prothrombin activation and this leads to a significant acceleration of CFT.

Although MASP-1 activates MASP-2 which, in turn, is able to promote clotting by prothrombin cleavage similar to FXa-mediated cleavage (Krarup et al., 2007), inhibition of MASP-2 in WB and PPP samples showed that MASP-2 does not further enhance the capability of MASP-1 to promote clotting.

We further demonstrated that MASP-1-mediated clot formation is depending on prothrombin. In PT-DP and a purified system, MASP-1 alone failed to induce clotting in the absence of prothrombin, and supplementation with prothrombin was able to reconstitute clotting. These experiments confirmed that MASP-1 induces clotting indirectly by activating prothrombin.

In the coagulation cascade, prothrombin is processed to thrombin by $\mathrm{FXa}$, giving rise to several intermediates exhibiting different functions (Krishnaswamy, 2013). Intriguingly, MASP-2 was shown to cleave prothrombin in a similar manner, whereas prothrombin cleavage by MASP-1 was not detected (Krarup et al., 2007). Here we show by SDS-PAGE analysis that MASP-1 is indeed capable of cleaving prothrombin. N-terminal sequencing of cleavage products revealed three cleavage sites on prothrombin, among which R393 is not cleaved by FXa, but it is a site of autodegradation by a-thrombin during prolonged incubation (Bovill et al., 1995). R393, as R320, is located between the interchain disulfide-bridge (C292-C438), therefore cleavage at R393 does not lead to dissociation of the heavy and light chains but presumably maintains the two-chain conformation and may give rise to an alternative active form of thrombin.

Similar to FXa-mediated prothrombin activation, several intermediate forms appear in the course of prothrombin digestion by MASP-1. In FXa-mediated prothrombin activation, the intermediate meizothrombin is enzymatically active and responsible for feedback-cleavage at position R155 of prothrombin which is also a cleavage site for a-thrombin (Krishnaswamy, 2013). Also in MASP-1- 
mediated prothrombin cleavage, intermediate forms and/or thrombin species contribute to further processing of prothrombin and its cleavage products. Upon inhibition of thrombin species by hirudin, the heavy chain of the putative alternative thrombin species (band $h$ ) does hardly appear. Thus, it is likely that a thrombin species cleaved at R320 and therefore inhibitable by hirudin must be involved. However, we were not able to isolate a fragment carrying the $\mathrm{N}$-terminal sequence of R320. An explanation for these findings could be that MASP-1 cleaves at both R320 and R393, yielding a thrombin form resembling $\beta$-thrombin or even $\beta$-thrombin itself (Bovill et al., 1995). This thrombin species could be responsible for the procoagulant action of MASP-1, as the appearance of the R393 heavy chain (band h) at approximately 40 min roughly coincides with clotting at 32 min in the purified system in thrombelastography.

Further work is ongoing to prove the existence and function of an alternative form of thrombin, and to clarify the chronological order of prothrombin degradation by MASP-1 with the help of prothrombin variants with mutated cleavage sites.

Our study may have (patho-) physiological implications. We have recently reported elevated MASP-1 levels in patients with myocardial infarction (Frauenknecht et al., 2013) and in patients with type-1 diabetes (Jenny et al., 2015). MASP-1 levels have also been shown to double during acute-phase reactions (Thiel et al., 2012). We therefore believe that MASP-1 levels are elevated and MASP-1 is activated in the proinflammatory and procoagulant environment of atherosclerosis and contributes to clot formation by activation of coagulation factors and endothelial cells (Dobó et al., 2014).

\section{Conclusion}

In summary, we have shown that MASP-1 is able to induce and promote clot formation in whole blood and plasma, measured in a global setting using the technique of thrombelastography. Experiments in PT-DP and a purified system confirmed that MASP-1-induced clotting is dependent on prothrombin, as MASP-1 lacks any clotting activity in the absence of prothrombin. Finally, we 
have demonstrated that MASP-1 cleaves prothrombin and identified its cleavage sites, suggesting that MASP-1 gives rise to an alternative active form of thrombin.

\section{Acknowledgements}

The authors would like to thank Prof. Johann Schaller and Urs Kämpfer (Department of Chemistry and Biochemistry, University of Bern) for their technical support and expertise in N-terminal protein sequencing, Dr. Andrea Kocsis (Institute of Enzymology, Budapest, Hungary) for the preparation of SGMI-2, and Dr. Mikkel-Ole Skjoedt (Copenhagen, Denmark) for the donation of full-length MASP1.

This work was supported by grants from the Swiss National Science Foundation (grant 140925), OPO Foundation (Zurich, Switzerland), Hungarian Scientific Research Fund (OTKA grant NK100834), and the János Bolyai Research Fellowship of the Hungarian Academy of Sciences.

\section{Author contributions}

L. Jenny designed and performed the experiments, analysed the data, and wrote the manuscript.

J. Dobó and P. Gál produced the rMASP-1 fragment and revised the manuscript. V. Schroeder designed the study, analysed the data, and revised the manuscript.

\section{Conflict of interest}

The authors have no competing interests. 


\section{References}

Amara, U., Flierl, M.A., Rittirsch, D., Klos, A., Chen, H., Acker, B., Brückner, U.B., Nilsson, B., Lambris, J.D., Huber-Lang, M., 2010. Molecular intercommunication between the complement and coagulation systems. J. Immunol. 185, 5628-5636.

Bovill, E.G., Tracy, R.P., Hayes, T.E., Jenny, R.J., Bhushan, F.H., Mann, K.G., 1995. Evidence that meizothrombin is an intermediated product in the clotting of whole blood. Arterioscler. Thromb. Vasc. Biol. 15, 754-758.

Brummel, K.E., Paradis, S.G., Butenas, S., Mann, K.G., 2002. Thrombin functions during tissue factor-induced blood coagulation. Blood 100, 148-152.

Dahl, M.R., Thiel, S., Matsushita, M., Fujita, T., Willis, A.C., Christensen, T., Vorup-Jensen, T., Jensenius, J.C., 2001. MASP-3 and its association with distinct complexes of the mannanbinding lectin complement activation pathway. Immunity 15, 127-135.

Dobó, J., Harmat, V., Beinrohr, L., Sebestyén, E., Závodszky, P., Gál, P., 2009. MASP-1, a promiscuous complement protease: structure of its catalytic region reveals the basis of its broad specificity. J. Immunol. 183, 1207-1214.

Dobó, J., Schroeder, V., Jenny, L., Cervenak, L., Závodszky, P., Gál, P., 2014. Multiple roles of complement MASP-1 at the interface of innate immune response and coagulation. Mol. Immunol. 61, 69-78.

Frauenknecht, V., Thiel, S., Storm, L., Meier, N., Arnold, M., Schmid, J.P., Saner, H. \& Schroeder, V., 2013. Plasma levels of mannan-binding lectin (MBL)-associated serine proteases (MASPs) and MBL-associated protein in cardio- and cerebrovascular diseases. Clin. Exp. Immunol. 173, 112-120.

Gál, P., Dobó, J., Závodszky, P., Sim, R.B., 2009. Early complement proteases: C1r, C1s and MASPs. A structural insight into activation and functions. Mol. Immunol. 46, 2745-2752.

Haynes, L.M., Bouchard, B.A., Tracy, P.B., Mann, K.G., 2012. Prothrombin activation by plateletassociated prothrombinase proceeds through the prethrombin-2 pathway via a concerted mechanism. J. Biol. Chem. 287, 38647-38655.

Héja, D., Kocsis, A., Dobó, J., Szilágyi, K., Szász, R., Závodszky, P., Pál, G., Gál, P., $2012 a$. Revised mechanism of complement lectin-pathway activation revealing the role of serine 
protease MASP-1 as the exclusive activator of MASP-2. Proc. Natl. Acad. Sci. USA., 109, 10498-10503.

Héja, D., Harmat, V., Fodor, K., Wilmanns, M., Dobó, J., Kékesi, K.A., Závodszky, P., Gál, P. \& Pál, G., 2012b. Monospecific inhibitors show that both mannan-binding lectin-associated serine protease-1 (MASP-1) and -2 are essential for lectin pathway activation and reveal structural plasticity of MASP-2. J. Biol. Chem. 287, 20290-20300.

Hess, K., Ajjan, R., Phoenix, F., Dobó, J., Gál, P., Schroeder, V., 2012. Effects of MASP-1 of the complement system on activation of coagulation factors and plasma clot formation. PLoS One 7, e35690.

Jenny, L., Ajjan, R., King, R., Thiel, S. \& Schroeder, V., 2015. Plasma levels of mannan-binding lectin-associated serine proteases MASP-1 and MASP-2 are elevated in type 1 diabetes and correlate with glycaemic control. Clin. Exp. Immunol., in press.

Krarup, A., Wallis, R., Presanis, J.S., Gál, P., Sim, R.B., 2007. Simultaneous activation of complement and coagulation by MBL-associated serine protease 2. PLoS One 2, e623.

Krarup, A., Gulla, K.C., Gál, P., Hajela, K., Sim, R.B., 2008. The action of MBL-associated serine protease 1 (MASP1) on factor XIII and fibrinogen. Biochim. Biophys. Acta 1784, 1294-1300.

Krem, M.M., Di Cera, E., 2002. Evolution of enzyme cascades from embryonic development to blood coagulation. Trends Biochem. Sci. 27, 67-74.

Krishnaswamy, S., 2013. The transition of prothrombin to thrombin. J. Thromb. Haemost. 11 (Suppl 1), 265-276.

La Bonte, L.R., Pavlov, V.I., Tan, Y.S., Takahashi, K., Takahashi, M., Banda, N.K., Zou, C., Fujita, T., Stahl G.L., 2012. Mannose-binding lectin-associated serine protease-1 is a significant contributor to coagulation in a murine model of occlusive thrombosis. J. Immunol. 188, 885891.

Mayilyan, K.R., Kang, Y.H., Dodds, A.W., Sim, R.B., 2008. The complement system in innate immunity, in: Heine, H., Innate Immunity of Plants, Animals and Humans. Springer, Berlin Heidelberg, pp. 219-236.

Megyeri, M., Makó, V., Beinrohr, L., Doleschall, Z., Prohászka, Z., Cervenak, L., Závodszky, P., Gál, P., 2009. Complement protease MASP-1 activates human endothelial cells: PAR4 
activation is a link between complement and endothelial function. J. Immunol. 183, 34093416.

Megyeri, M., Harmat, V., Major, B., Végh, Á., Balczer, J., Héja, D., Szilágyi, K., Datz, D., Pál, G., Závodszky, P., Gál, P., Dobó, J., 2013. Quantitative characterization of the activation steps of mannan-binding lectin (MBL)-associated serine proteases (MASPs) points to the central role of MASP-1 in the initiation of the complement lectin pathway. J. Biol. Chem. 288, 89228934.

Oikonomopoulou, K., Ricklin, D., Ward, P.A., Lambris, J.D., 2012. Interactions between coagulation and complement - their role in inflammation. Semin. Immunopathol. 34, 151165.

Parej, K., Dobó, J., Závodszky, P., Gál, P., 2013. The control of the complement lectin pathway activation revisited: both C1-inhibitor and antithrombin are likely physiological inhibitors, while a2-macroglobulin is not. Mol. Immunol. 54, 415-422.

Parry, M.A., Stone, S.R., Hofsteenge, J., Jackman, M.P., 1993. Evidence for common structural changes in thrombin induced by active-site or exosite binding. Biochem. J. 290, 665-670.

Pavlov, V.I., Skjoedt, M.O., Siow Tan, Y., Rosbjerg, A., Garred, P., Stahl, G.L., 2012. Endogenous and natural complement inhibitor attenuates myocardial injury and arterial thrombogenesis. Circulation. 126, 2227-2235.

Presanis, J.S., Hajela, K., Ambrus, G., Gál, P., Sim, R.B., 2003. Differential substrate and inhibitor profiles for human MASP-1 and MASP-2. Mol. Immunol. 40, 921-929.

Ricklin, D., Hajishengallis, G., Yang, K., Lambris, J.D., 2010. Complement: a key system for immune surveillance and homeostasis. Nat. Immunol. 11, 785-97.

Stover, C.M., Thiel, S., Thelen, M., Lynch, N.J., Vorup-Jensen, T., Jensenius, J.C., Schwaeble, W.J., 1999. Two constituents of the initiation complex of the mannan-binding lectin activation pathway of complement are encoded by a single structural gene. J. Immunol. 162, 3481-3490.

Takahashi, K., Chang, W.C., Takahashi, M., Pavlov, V., Ishida, Y., La Bonte, L., Shi, L., Fujita, T., Stahl, G.L., Van Cott, E.M., 2011. Mannose-binding lectin and its associated proteases (MASPs) mediate coagulation and its deficiency is a risk factor in developing complications 
from infection, including disseminated intravascular coagulation. Immunobiology 216, 96102.

Thiel, S., Jensen, L., Degn, S.E., Nielsen, H.J., Gál, P., Dobó, J., Jensenius, J.C., 2012. Mannanbinding lectin (MBL)-associated serine protease-1 (MASP-1), a serine protease associated with humoral pattern-recognition molecules: normal and acute-phase levels in serum and stoichiometry of lectin pathway components. Clin. Exp. Immunol. 169, 38-48.

Yongqing, T., Drentin, N., Duncan, R.C., Wijeyewickrema, L.C., Pike, R.N., 2012. Mannose-binding lectin serine proteases and associated proteins of the lectin pathway of complement: two genes, five proteins and many functions? Biochim. Biophys. Acta 1824, 253-262. 
Table

Table 1. Clot formation parameters in PPP, PT-DP or a purified system.

\begin{tabular}{|c|c|c|c|c|c|c|c|}
\hline System & $\begin{array}{c}\text { MASP-1 } \\
\mu \mathrm{g} / \mathrm{ml}(\mathrm{nmol} / \mathrm{l})\end{array}$ & $\begin{array}{c}\text { Thrombin } \\
\mu \mathrm{g} / \mathrm{ml}(\mathrm{nmol} / \mathrm{l})\end{array}$ & $\begin{array}{c}\text { Fibrinogen } \\
\mathrm{mg} / \mathrm{ml}(\mu \mathrm{mol} / \mathrm{l})\end{array}$ & $\begin{array}{c}\text { Prothrombin } \\
\mu \mathrm{g} / \mathrm{ml}(\mu \mathrm{mol} / \mathrm{l})\end{array}$ & $\begin{array}{c}\mathbf{C T} \\
{[\mathrm{s}]}\end{array}$ & $\begin{array}{c}\text { CFT } \\
{[\mathrm{s}]}\end{array}$ & $\begin{array}{c}\text { MCF } \\
{[\mathrm{mm}]}\end{array}$ \\
\hline PPP & $10(220)$ & - & - & - & 948 & 356 & 34 \\
\hline PPP & - & $0.74(20)$ & - & - & 58 & 389 & 36 \\
\hline PT-DP & $80(1760)$ & - & - & - & - & - & - \\
\hline PT-DP & - & $0.74(20)$ & - & - & 122 & $11^{\prime} 811$ & 20 \\
\hline PT-DP & $80(1760)$ & - & - & $100(1.4)$ & 279 & 1394 & 23 \\
\hline Purified & $80(1760)$ & - & $2(5.8)$ & - & - & - & - \\
\hline Purified & - & $0.74(20)$ & $2(5.8)$ & - & 174 & - & 15 \\
\hline Purified & $80(1760)$ & - & $2(5.8)$ & $100(1.4)$ & 17741 & $22^{\prime} 016$ & 32 \\
\hline
\end{tabular}

Protein concentrations used are shown in mass and molar concentrations. Thrombelastographic parameters are shown as mean values $(n=3)$. PPP $=$ platelet-poor plasma. PT-DP= prothrombindepleted plasma. $\mathrm{CT}=$ clotting time. $\mathrm{CFT}=$ clot formation time $\mathrm{MCF}=$ maximum clot firmness. 


\section{Figure legends}

Figure 1. Effects of rMASP-1cf on clotting time (CT) measured by thrombelastometry. Dosedependent effects of rMASP-1cf, thrombin or combinations of both enzymes on the clotting time (CT) measured by ROTEM® in (A) whole blood (WB) and (B) platelet-poor plasma (PPP). Results are expressed relative to recalcified-only samples labelled as control. Enzyme concentrations are in nanomolar. Error bars represent the standard deviation of six measurements.

Figure 2. Effects of rMASP-1cf on clot formation time (CFT) measured by thrombelastometry. Dose-dependent effects of rMASP-1cf, thrombin or combinations of both enzymes on the clot formation time (CFT) measured by ROTEM® in (A) whole blood (WB) and (B) platelet-poor plasma (PPP). Results are expressed relative to recalcified-only samples labelled as control. Enzyme concentrations are in nanomolar. Error bars represent the standard deviation of six measurements.

Figure 3. Time-course of the digestion of prothrombin by MASP-1. (A) rMASP-1cf and prothrombin were incubated for up to $90 \mathrm{~min}$ (shown in the first lane per time point). Hirudin was added to abolish effects of thrombin species (shown in the second lane per time point). Bands were identified as a) uncleaved prothrombin, b) prethrombin-1, c) "meizothrombin analogue" cleaved at R393, d) prethrombin-2, e) fragment F1.F2, f) MASP-1 heavy chain, g) degradation product of the MASP-1 heavy chain, $h$ ) shortened version of the $\alpha$-thrombin heavy chain (cleaved at R393), i) MASP-1 light chain, j) fragment F1. (B) The control gel shows prothrombin, prothrombin (90 min incubation), rMASP-1cf (heavy and light chain), hirudin, prothrombin/thrombin incubation (for $90 \mathrm{~min}$ ) and prothrombin/FXa incubation (90 min). The bands correspond to: I) prothrombin, II) prethrombin-1, III) prethrombin-2, IV) fragment F1.F2, V) thrombin heavy chain.

Figure 4. Cleavage sites in prothrombin. Prothrombin domains are fragment 1 (F1), fragment 2 (F2), thrombin light chain (LC), and thrombin heavy chain (HC). LC and HC are connected by a disulfide bond. All cleavage sites occur at arginine residues (R). Numbering of amino acids starts 
at the first amino acid of F1. Identified cleavage sites for rMASP-1cf: R155, also cleaved by thrombin/meizothrombin. R271, also cleaved by FXa. R393, cleavage site of MASP-1. Cleavage by $\mathrm{FXa}$ at R320 between the light and heavy chain of thrombin leads to its activation. 
Figure 1

A

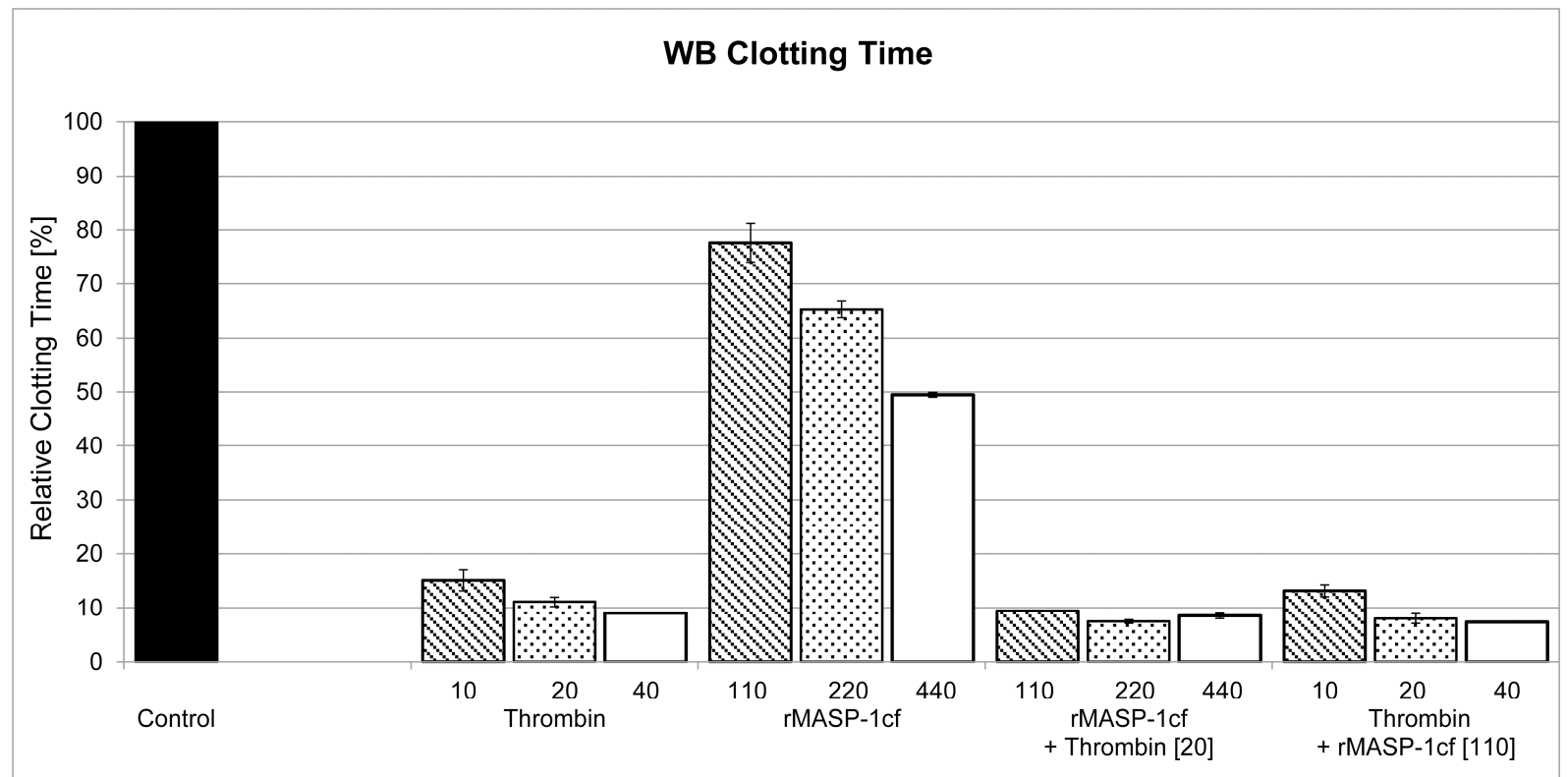

B

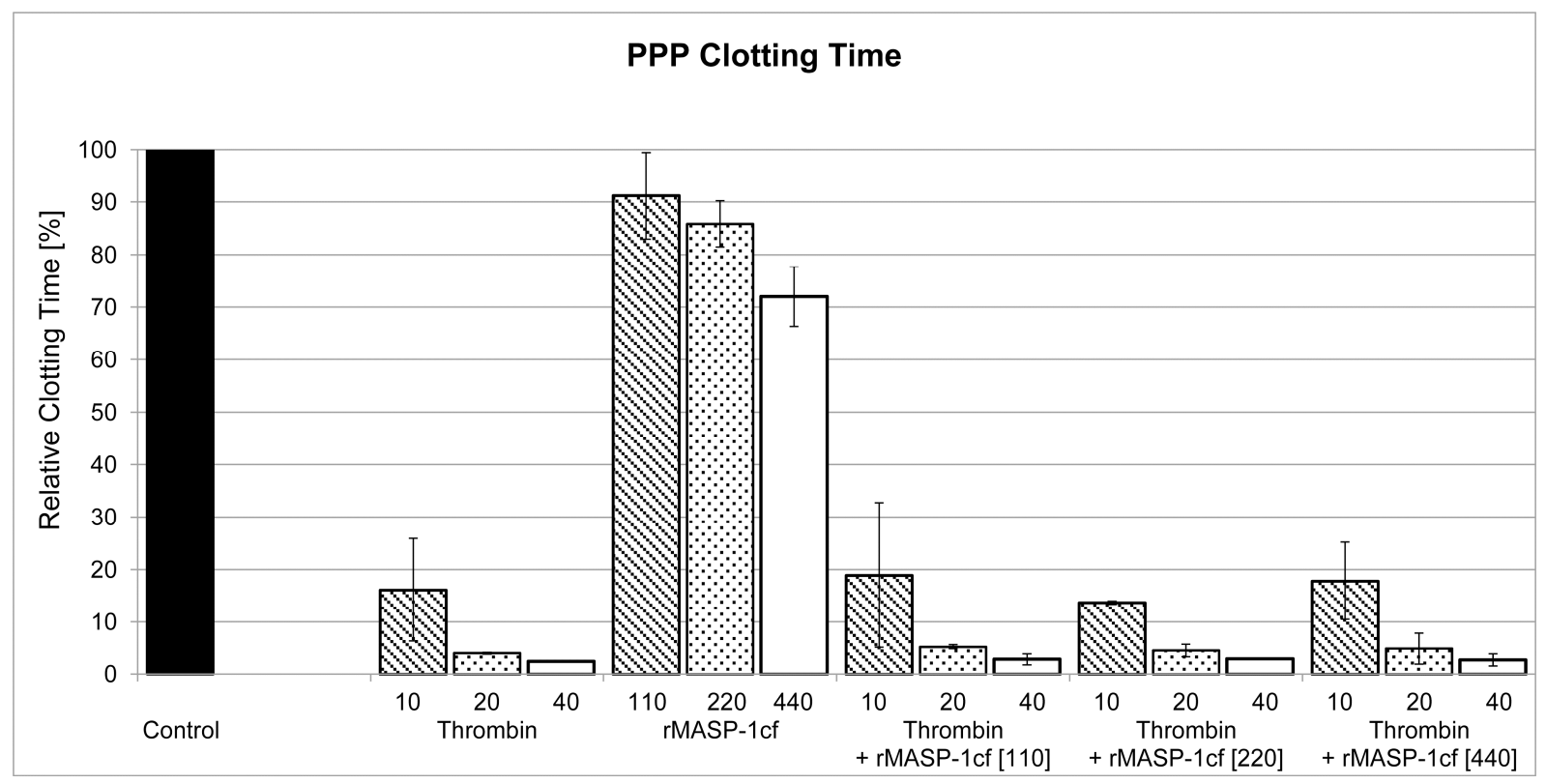


Figure 2

A

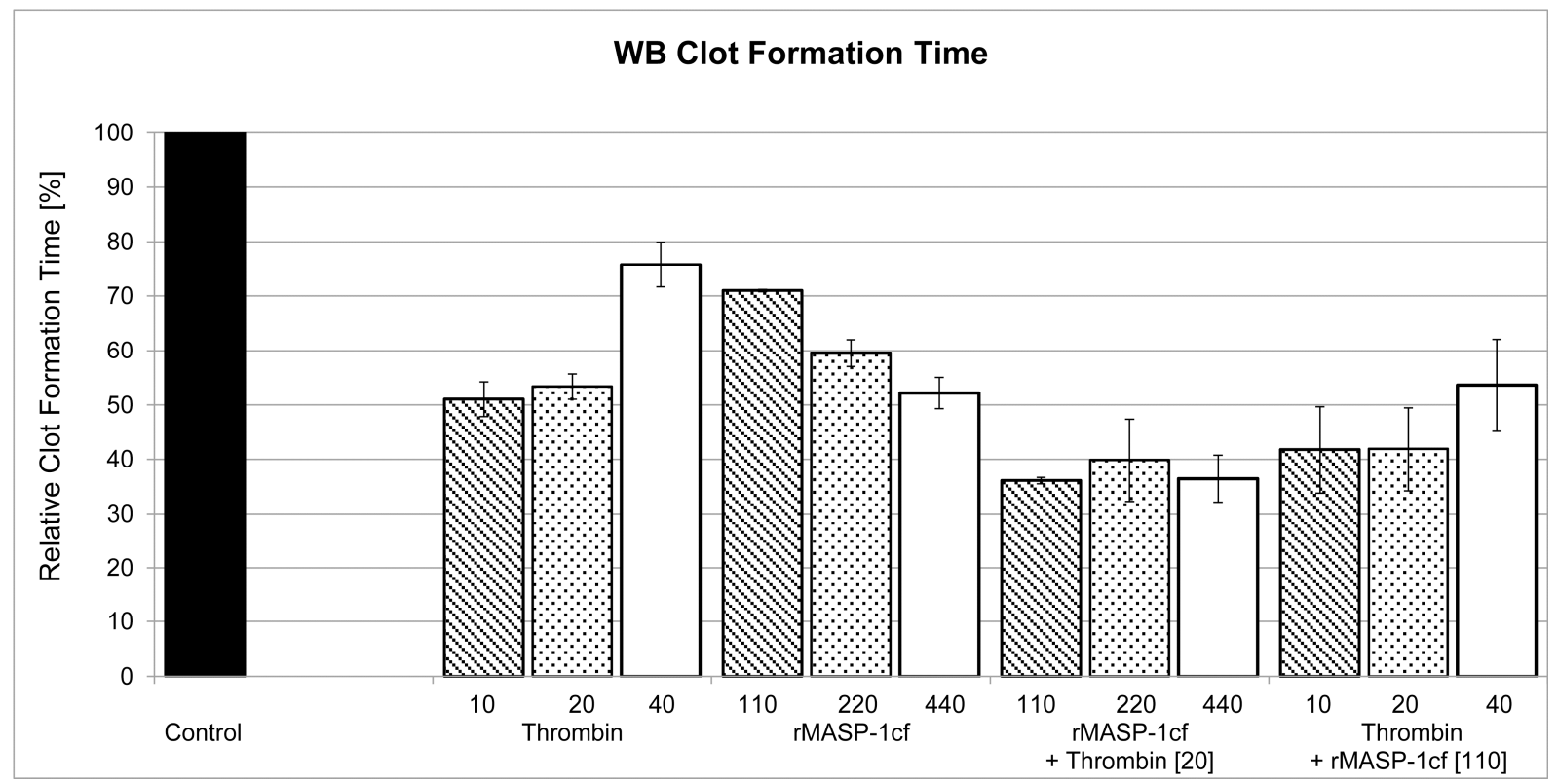

B

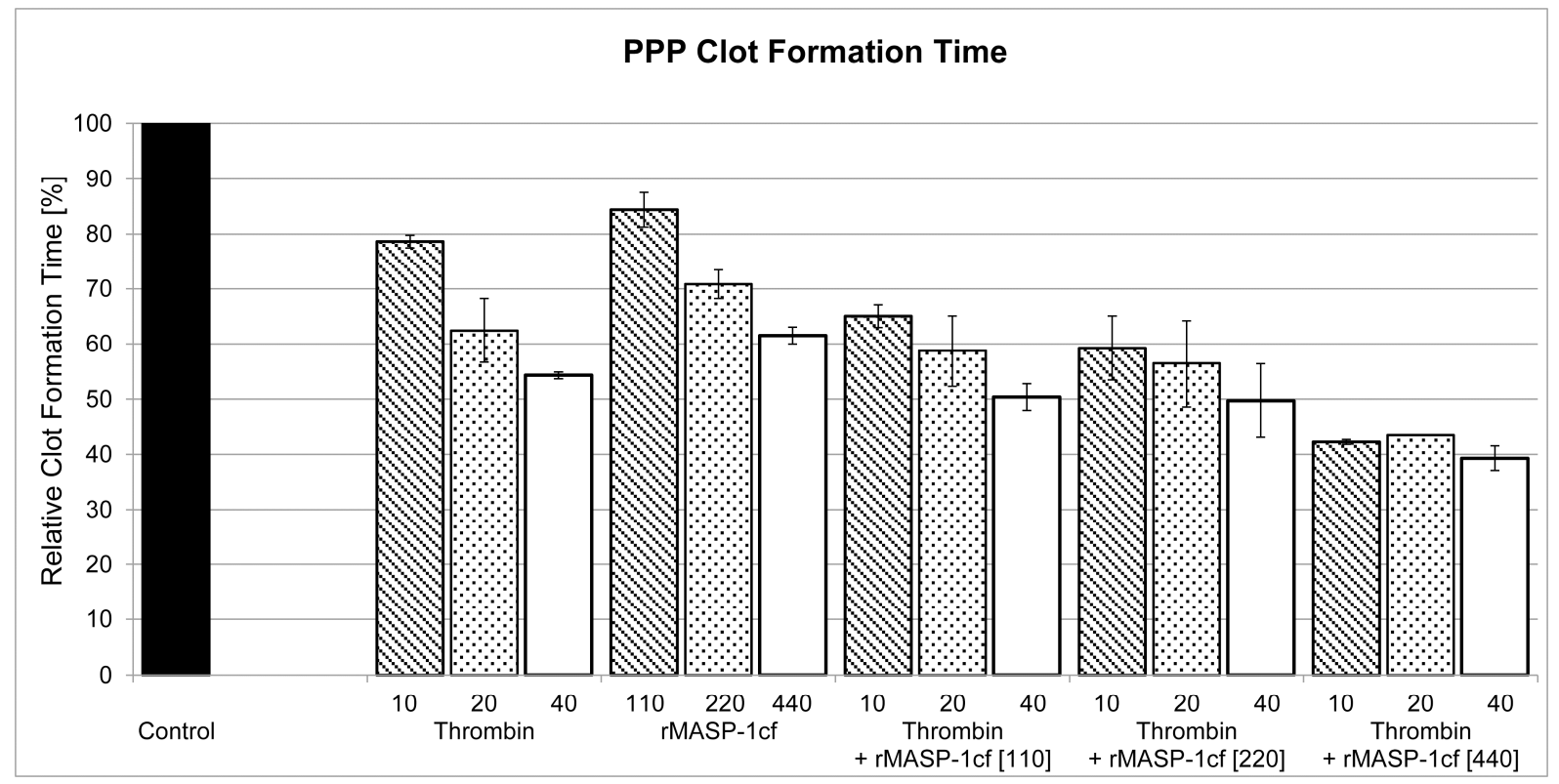


Figure 3

A
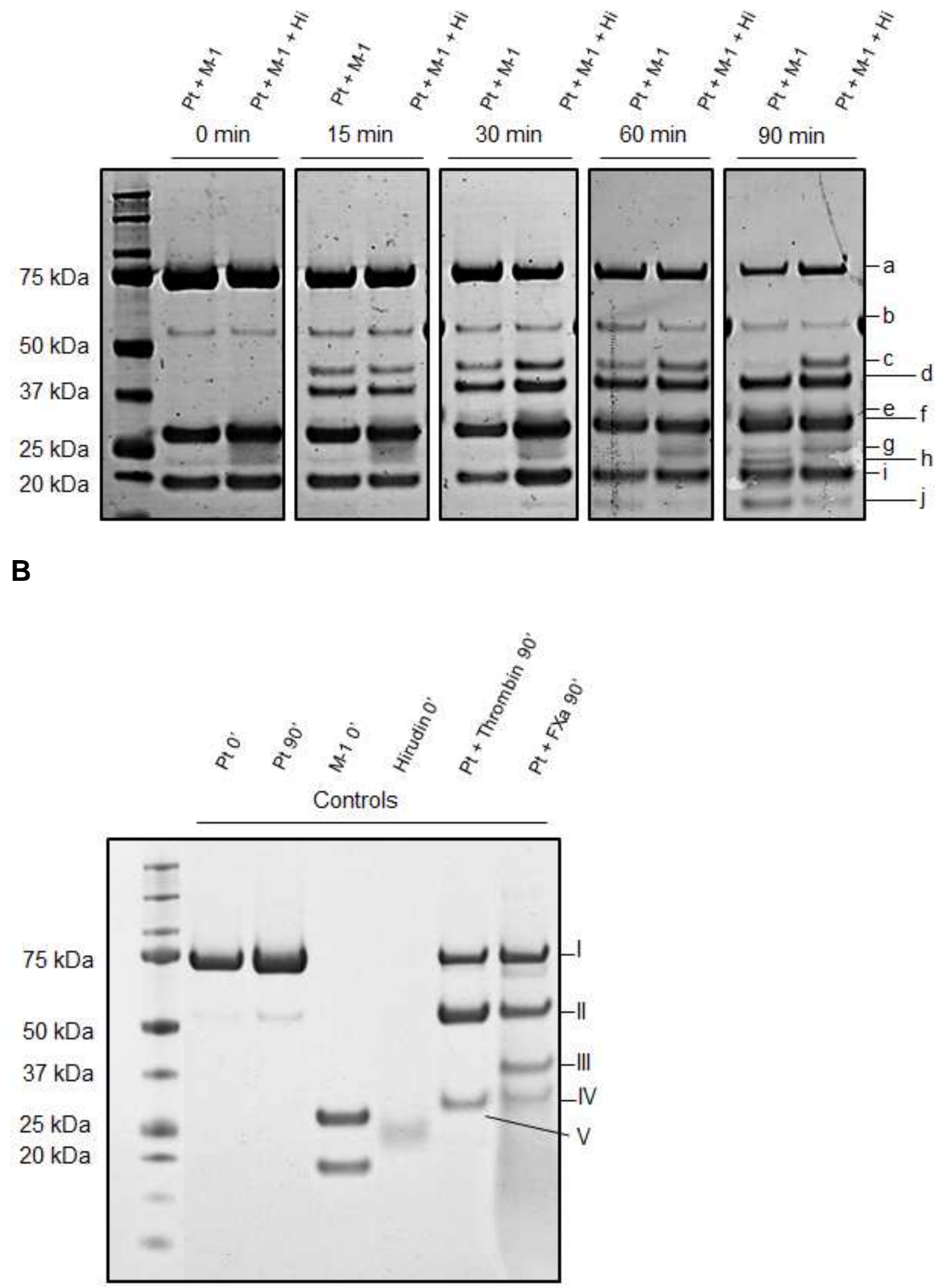
Figure 4

\section{Prothrombin}

\begin{tabular}{|c|c|c|c|}
\hline F1 & F2 & LC & $\mathrm{HC}$ \\
\hline
\end{tabular}


Figure S1. Overlay of thrombelastographic graphs of PPP and WB incubated in presence or absence of full-length MASP-1

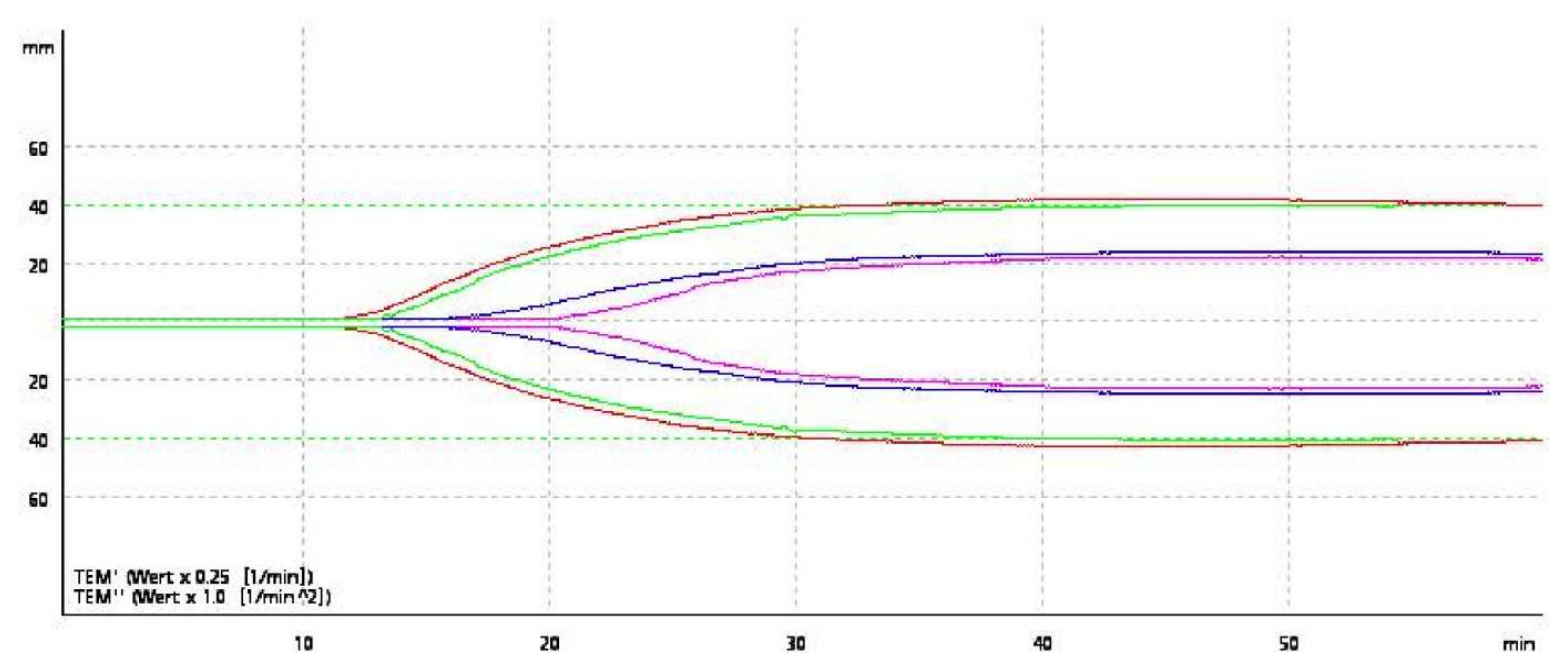

The $\mathrm{X}$-axis depicts the time in minutes, whereas the $\mathrm{Y}$-axis shows the clot firmness in $\mathrm{mm}$. Red: WB incubated with full-length MASP-1. Green: WB incubated without full-length MASP-1. Blue: PPP incubated with full-length MASP-1. Pink: PPP incubated without full-length MASP-1.

Table S1. Clot formation parameters in PPP and WB with or without incubation of full-length MASP-1.

\begin{tabular}{|c|c|c|c|c|}
\hline System & $\begin{array}{c}\text { Full length MASP-1 } \\
\mu \mathrm{g} / \mathrm{ml}(\mathrm{nmol} / \mathrm{l})\end{array}$ & $\mathbf{C T}[\mathrm{s}]$ & CFT [s] & MCF [mm] \\
\hline PPP & - & 1224 & 811 & 23 \\
\hline PPP & $20(260)$ & 962 & 781 & 25 \\
\hline WB & - & 698 & 350 & 43 \\
\hline WB & $20(260)$ & 783 & 341 & 41 \\
\hline
\end{tabular}

Protein concentrations used are shown in mass and molar concentrations. Thrombelastographic parameters are shown as mean values. $\mathrm{PPP}=$ platelet-poor plasma. $\mathrm{PT}-\mathrm{DP}=$ prothrombin-depleted plasma. $\mathrm{CT}=$ clotting time. $\mathrm{CFT}=$ clot formation time. $\mathrm{MCF}=$ maximum clot firmness. 